since in $\mathrm{e}^{(\boldsymbol{F})}(1)$ the matrix element must be antisymmetric under interchange of $q_{1}$, and $q_{2}$. Therefore the limit of $O^{(B)}$, and also of $\mathfrak{H}^{(\mathrm{B})}$, does not exist, and no inconsistency occurs.

\section{SUMMARY}

We can summarize this situation by saying that in the high- $p$ limit a spin-0 para-Bose field has (a) annihilation and creation operators and an anti- commutator which approach the annihilation and creation operators and $c$-number anticommutator of a spin-0 Fermi field and (b) a Hamiltonian and a set of number operators which approach those of the spin-0 Fermi field, but that, on the contrary, the Hamiltonian density of the high-order para-Bose theory does not converge to an operator in the Fermi theory. Analogous statements hold for the high- $p$ limit of a spin- $\frac{1}{2}$ para-Fermi field.

\title{
Statistical Mechanics of Assemblies of Coupled Oscillators*
}

\author{
G. W. Fond \\ Department of Physics, University of Michigan, Ann Arbor, Michigan \\ M. $\mathrm{K}_{\mathrm{AC}}$ \\ The Rockefeller Institute, New York, New York \\ AND \\ P. Mazur \\ Lorentz Institute for Theoretical Physics, Leiden, The Netherlands \\ (Received 25 September 1964)
}

\begin{abstract}
It is shown that a system of coupled harmonic oscillators can be made a model of a heat bath. Thus a particle coupled harmonically to the bath and by an arbitrary force to a fixed center will (in an appropriate limit) exhibit Brownian motion. Both elassical and quantum mechanical treatments are given.
\end{abstract}

\section{INTRODUCTION}

$\mathrm{O}$ UR aim here is to study a simple mechanical model, a chain of coupled harmonic oscillators, in order to come to a deeper understanding of some of the phenomena associated with Brownian motion. ${ }^{1,2}$ With this model we are able to carry through the program one would like to achieve with more

* Part of the work reported in this paper was done while one of us (M. K.) was H. A. Lorentz Visiting Professor at the University of Leiden. When this work was reported at Yeshiva University in the Fall of 1963 Dr. N. L. Balazs informed us that he had independently obtained some of the results concerning the classical case.

$t$ Supported in part by a grant from the National Science Foundation.

1 There is an extensive literature on the motion of coupled oscillators, mostly concerned with motion in a lattice with nearest-neighbor interactions. Some of the more recent articles which have a bearing on our work are: $P$. Mazur and E. Montroll, J. Math. Phys. 1, 70 (1960); P. C. Hernmer, "Dynamic and Stochastic Types of Motion in the Linear Chain," thesis, Norges Tekniske Høgskole, Trondheim, Norway (1959); R. J. Rubin, J. Math. Phys. 1, 309 (1960); 2, 373 (1961); M. Toda and Y. Koguri, Suppl. Progr. Theoret. Phys. (Kyoto) 23, 157 (1962); R. E. Turner, Physica 26, $274(1960)$.

2 The classic papers on the phenomological theory of Brownian motion are: G. E. Uhlenbeck and L. S. Ornstein, Phys. Rev. 36, 823 (1930); M. C. Wang and G. E. Uhlenbeck, Rev. Mod. Phys. 17, 323 (1945). realistic interactions. This program, which really goes back to Gibbs, goes as follows ${ }^{3}$ :

(i) Solve the equations of motion of the mechanical system consisting of a Brownian particle coupled to heat bath. The solution consists of expressions for the coordinates and momenta at time $t$ in terms of the initial coordinates and momenta.

(ii) Assume the initial coordinates and momenta of the heat bath to be distributed according to some statistical distribution, e.g., that of the canonical ensemble.

(iii) Show that the coordinate and momentum of the Brownian particle, as functions of time, will then represent stochastic processes (whose statistical properties arise from the initial distribution of the heat bath) of the kind familiar from standard theories.

This is a very ambitious program, and it is no wonder that it can be carried out only for the simplest models.

${ }^{3}$ See the article by H. Wergeland in Fundamental Problems in Statistical Mechanics, edited by E. G. D. Cohen (NorthHolland Publishing Company, Amsterdam, 1962). 
We have a good idea of what the results of this program should be, since, after all, Brownian motion is a throughly studied experimental phenomenon with a satisfactory phenomenological theory. In general we expect to show:

(i) The approach to equilibrium. In particular, the distribution of momentum of the Brownian particle should approach the Maxwellian distribution.

(ii) The description of this approach to equilibrium should be contracted, i.e., should involve only a small number of the possible variables describing the system. Another way of saying this is that there should be a reduced description in terms of which the stochastic process is Markoffian.

(iii) What changes occur when one adopts a quantum description of the system. Here we have less of an idea of what we should expect, but somehow the basic features of the stochastic process should be preserved in the quantum description.

We can be still more explicit about what we mean by a contracted description of Brownian motion; we mean the Langevin equation of motion. For a Brownian particle of mass $m$ acted upon by an outside force $F(x)$ this equation is

$$
\dot{p}=-f p / m+E(t)+F(x),
$$

where $p=m \dot{x}$ is the momentum of the Brownian particle, $f$ the friction constant, and $E(t)$ is the random force due to the heat bath. This random force is a purely random Gaussian process characterized by

$$
\langle E(t)\rangle=0, \quad\left\langle E(t) E\left(t^{\prime}\right)\right\rangle=2 f k T \delta\left(t-t^{\prime}\right),
$$

where $T$ is the temperature of the heat bath and $k$ is Boltzmann's constant. Note that the Langevin equation is a contracted description in the sense that the heat bath is described by only two parameters, the friction constant and the temperature, and that only the first two time derivatives of the position $x$ of the Brownian particle appear. ${ }^{4}$

In Sec. 2 we discuss the dynamics of a system of coupled oscillators. There we formally carry through the program for the case of an arbitrary coupling of the oscillators. In Sec. 3 the arbitrary coupling of a linear chain is considered, and we show that there is a coupling for which, in the limit of an infinite chain, the resulting stochastic process is Markoffian. Taking the chain of oscillators with this coupling as the heat bath, we derive in Sec. 4 the Langevin equation for a Brownian particle. In Sec. 5 we discuss the quantum description of the system, and in Sec. 6 we discuss the quantum Langevin

1 That is, there are no memory effects. equation. Finally, in Sec. 7 we consider the Brownian motion of a quantum oscillator.

\section{DYNAMICS OF A SYSTEM OF COUPLED OSCILLATORS}

Consider a system of $(2 N+1)$ coupled oscillators with Hamiltonian:

$$
H=\frac{1}{2} \sum_{i=-N}^{N} p_{i}^{2}+\frac{1}{2} \sum_{i, k=-N}^{N} q_{i} A_{i k} q_{k} .
$$

Here $q_{i}$ and $p_{i}$ are, respectively, the canonical coordinate and momentum of the $j$ th oscillator. The mass of each oscillator has been taken to be unity. The interactions of the oscillators are characterized by the $(2 N+1) \times(2 N+1)$ symmetric matrix A, whose elements are the $A_{i k}$. At present, we make no special assumptions about this matrix except that it has no negative eigenvalues. The canonical equations of motion may be conviently written in matrix notations as follows:

$$
\dot{\mathbf{q}}=\mathbf{p}, \quad \dot{\mathbf{p}}=-\mathbf{A q} .
$$

Here $p$ and $q$ are $(2 N+1)$-rowed column matrices whose elements are the $p_{i}$ and $q_{i}$, respectively. The formal solution of the equations of motion is

$$
\begin{aligned}
& \mathbf{q}(t)=\cos \left(\mathbf{A}^{\frac{1}{2}} t\right) \mathbf{q}(0)+\mathbf{A}^{-\frac{1}{2}} \sin \left(\mathbf{A}^{\frac{1}{2}} t\right) \mathbf{p}(0), \\
& \mathbf{p}(t)=-\mathbf{A}^{\frac{1}{3}} \sin \left(\mathbf{A}^{\frac{1}{2}} t\right) \mathbf{q}(0)+\cos \left(\mathbf{A}^{\frac{1}{2}} t\right) \mathbf{p}(0),
\end{aligned}
$$

where, e.g.,

$$
\cos \mathbf{A}^{\frac{1}{1}} t=\sum_{n=0}^{\infty} \frac{(-)^{n}}{(2 n) !} \mathbf{A}^{n} t^{2 n}
$$

We now assume that at $t=0$ the system is in equilibrium at temperature $T$. That is, we assume that the $q_{i}(0)$ and $p_{i}(0)$ are distributed according to the canonical distribution

$$
D(\mathbf{q}(0), \mathbf{p}(0))=(2 \pi / \beta)^{2 N+1}(\operatorname{det} \mathbf{A})^{-\frac{1}{1}} e^{-\beta H(q(0), p(0))},
$$

where $\beta=(k T)^{-1}$ and $\operatorname{det} \mathbf{A}$ is the determinant of $\mathbf{A}$. Note that there is a difficulty here, since $\operatorname{det} \mathbf{A}=0$ if $\mathbf{A}$ has zero eigenvalues. We therefore assume for the time being that $\mathbf{A}$ has no zero eigenvalues. The expectation of any function $F(q(0), p(0))$ is given by

$$
\begin{array}{r}
\langle F\rangle=\int \cdots \int d q_{-N}(0) \cdots d q_{N}(0) d p_{-N}(0) \cdots d p_{N}(0) \\
\times F(\mathbf{q}(0), \mathbf{p}(0)) D(\mathbf{q}(0), \mathbf{p}(0)) .
\end{array}
$$

Now we ask, what are the properties of the stochastic variables $q_{i}(t)$ and $p_{i}(t)$ which result from (4) under the distribution (6)? First of all, it is clear that the process is Gaussian. This follows from the fact that the distribution (6) is Gaussian and that the relation (4) is linear. That the process is sta- 
tionary follows from the Liouville theorem of mechanies, which states that

$$
D(\mathbf{q}(t), \mathbf{p}(t))=D(\mathbf{q}(0), \mathbf{p}(0)) .
$$

It is well known that the statistical properties of such a stationary Gaussian process are completely described by the pair correlation functions. In our case these are obtained in Appendix 1; the results are ${ }^{5}$

$$
\begin{aligned}
& \left\langle p_{i}(t) p_{k}(t+\tau)\right\rangle=k T\left\|\cos \mathbf{A}^{\frac{1}{2}} \tau\right\|_{j k}, \\
& \left\langle q_{i}(t) p_{k}(t+\tau)\right\rangle=-k T\left\|\mathbf{A}^{-\frac{1}{2}} \sin \mathbf{A}^{\frac{1}{2}} \tau\right\|_{i k}, \\
& \left\langle q_{i}(t) q_{k}(t+\tau)\right\rangle=k T\left\|\mathbf{A}^{-1} \cos \mathbf{A}^{\frac{1}{2}} \tau\right\|_{i k} .
\end{aligned}
$$

Note that the position correlation (9c) involves the inverse of $\mathbf{A}$, which does not exist if $\mathbf{A}$ has zero eigenvalues.

If we fix our attention on a single oscillator, say the one with index 0 , the momentum autocorrelation is

$$
\left\langle p_{0}(t) p_{0}(t+\tau)\right\rangle=k T\left\|\cos \mathbf{A}^{\frac{3}{3}} \tau\right\|_{00} .
$$

This is the autocorrelation of a stationary Gaussian process in one variable. It is well known that such a process is Markoffian if and only if the autocorrelation is an exponential, i.e.,

$$
\left\langle p_{0}(t) p_{0}(t+\tau)\right\rangle=k T e^{-f|\tau|},
$$

where $f$ is a positive constant. The question we turn to in the next section is that of finding an interaction matrix A for which (10) assumes the form (11).

\section{THE INTERACTION MATRIX}

In our model we assume the $(2 N+1)$ oscillators are identical and that they are arranged in a chain with cyclic boundary conditions. This means that the interaction matrix $A$ is a symmetric cyclic matrix. ${ }^{6}$ The elements of such a matrix can be written in the form

$$
\begin{aligned}
A_{m x}=\frac{1}{2 N+1} \sum_{k=-N}^{N} \omega_{k}^{2} \\
\quad \times \exp \left\{i \frac{2 \pi}{2 N+1} k(m-n)\right\},
\end{aligned}
$$

where the symmetry of $A$ requires

$$
\omega_{k}^{2}=\omega_{-k}^{2} .
$$

The eigenvalues of this matrix are the quantities $\omega_{s}^{2}, s=-N,-N+1, \cdots, N$. That is

$$
A \xi^{(s)}=\omega_{s}^{2} \xi^{(s)} \text {, }
$$

\footnotetext{
${ }^{5}$ We use the notation $\|\mathbf{M}\|_{j k}$ for the element in the $j$ th row and $k$ th column of a matrix $\mathbf{M}$.

- See e. G. Kowalewski, Determinantentheorie (Chelsea Publishing Company, New York, 1948), 3rd ed., p. 105.
}

where the eigenvector $\xi^{(3)}$ is a $(2 N+1)$-rowed column matrix whose elements are

$$
\xi_{n}^{(s)}=(2 N+1)^{-\frac{1}{2}} \exp \{i[2 \pi /(2 N+1)] s n\} .
$$

These properties follow from the elementary formula

$$
\begin{aligned}
\sum_{k=-N}^{N} \exp \left(i k(m-n) \frac{2 \pi}{2 N+1}\right) & =\delta_{m, n}, \\
-N & \leq m, n \leq N .
\end{aligned}
$$

With this formula we can also readily demonstrate that if $F(\mathrm{~A})$ is a function of the matrix $\mathrm{A}$, then

$$
\begin{aligned}
\|F(\mathbf{A})\|_{m, n}= & \frac{1}{2 N+1} \sum_{k=-N}^{N} F\left(\omega_{k}^{2}\right) \\
& \times \exp \left\{i \frac{2 \pi}{2 N+1} k(m-n)\right\} .
\end{aligned}
$$

Note, incidentally, that the special case of nearestneighbor interactions is that for which

$$
\omega_{s}^{2}=\bar{\omega}^{2} \sin ^{2}[\pi s /(2 N+1)] .
$$

Consider next the limit $N \rightarrow \infty$, the infinite chain. If we make the additional assumption that $\omega_{0}^{2}$ is slowly varying function of $s$, then (12) becomes

$$
\begin{aligned}
A_{m n} & =\frac{1}{2 \pi} \int_{-\pi}^{\pi} d \theta f(\theta) e^{i(m-n) \theta} \\
& =\frac{1}{2 \pi} \int_{-\pi}^{\pi} d \theta f(\theta) \cos (m-n) \theta,
\end{aligned}
$$

where

$$
f(\theta)=\left\{\omega_{\theta}^{2}\right\}_{\alpha-(2 N+1) \theta / 2 \pi} .
$$

The relation (17) becomes in this limit

$$
\|F(\mathbf{A})\|_{m, n}=\frac{1}{2 \pi} \int_{-\pi}^{\pi} d \theta F(f(\theta)) \cos (m-n) \theta .
$$

We are now ready to turn to the problem posed at the end of the last section; that of finding an interaction matrix $A$ for which

$$
\left\|\cos \mathbf{A}^{\frac{1}{2}} t\right\|_{00}=e^{-f|t|} \text {. }
$$

Using the result (17) we see that for a finite matrix

$$
\left\|\cos \mathbf{A}^{\frac{1}{t}} t\right\|_{00}=\frac{1}{2 N+1} \sum_{k=-N}^{N} \cos \omega_{k} t .
$$

For any choice of the $\omega_{k}$ this is a quasiperiodic function and cannot be of the form (22). However, in the limit of large $N$, we can use (21) which gives

$$
\left\|\cos \mathbf{A}^{\frac{1}{2}} t\right\|_{00}=\frac{1}{2 \pi} \int_{-\pi}^{\pi} d \theta \cos \left\{[f(\theta)]^{\dagger} t\right\} .
$$

Taken with our requirement (22), this becomes an integral equation for $f(\theta)$. The answer is essentially 
unique and is ${ }^{7}$

$$
f(\theta)=f^{2} \tan ^{2} \frac{1}{2} \theta .
$$

There is a difficulty here, however; when (25) is inserted in the expression (19) for the matrix elements, the expression diverges! What we must do is employ a second limiting process (after the limit $N \rightarrow \infty)$, defining

$$
f_{\omega_{L}}(\theta)= \begin{cases}f^{2} \tan ^{2} \frac{\theta}{2}, & |\theta|<\theta_{L}, \\ 0, & \theta_{\mathrm{L}} \leq|\theta| \leq \pi .\end{cases}
$$

Here

$$
\omega_{\mathrm{L}} \equiv f \tan \frac{1}{2} \theta_{L}
$$

is a high-frequency cutoff in the spectrum of eigenfrequencies which ensures that the matrix elements (19) are finite. This frequency cutoff corresponds to a "microscopic interaction time" $\omega_{\mathrm{L}}^{-1}$ which we assume is very small compared with the "macroscopic relaxation time" $f^{-1}$. The result (22) holds strictly only in the limit $\omega_{\mathrm{L}} \rightarrow \infty$. Alternatively, we can say that, for $\omega_{\mathrm{L}} \gg f$ the result (22) holds for times long compared with $\omega_{\mathrm{L}}^{-1}$.

Our model, then, is that the interaction matrix elements are given by (19) with $f(\theta)$ given by (26) with $\omega_{\mathrm{L}} \gg f$. If in (24) we make the change of variable $\omega=f \tan \frac{1}{2} \theta$, we find

$$
\left\|\cos \mathbf{A}^{\frac{1}{2}} t\right\|_{00}=\frac{1}{\pi} \int_{-\omega_{L}}^{\omega_{L}} d \omega \frac{f}{\omega^{2}+f^{2}} \cos \omega t .
$$

In the limit $\omega_{L} \rightarrow \infty$ this becomes $e^{-f / t \mid}$ and, therefore, the Gaussian process $p_{0}(t)$ becomes also Markoffian.

\section{THE LANGEVIN EQUATION}

Having seen that our model leads to a Gaussian Markoffian stochastic process for the collection of coupled oscillators, we are led to ask whether it also leads to the Langevin equation for the motion of a single particle coupled to a heat bath consisting of such oscillators. In this section we see that this is indeed the case.

We select from the chain of $(2 N+1)$ oscillators, the particle with index 0 to be the Brownian particle; the remaining $2 N$ oscillators represent the heat bath. The outside force on this particle we denote by

$$
F(t) \equiv F\left(q_{0}(t)\right)
$$

If we define $\mathbf{F}(t)$ to be a $(2 N+1)$-rowed column

\footnotetext{
${ }^{7}$ What is unique is the spectrum of eigenfrequencies: $g(\omega)=2 \omega /\left(\pi f^{\prime}(\theta)\right)$, where $\theta$ is the function of $\omega$ obtained by inverting the equation $\omega^{2}=f(\theta)$. For (25), $g(\omega)=(2 f / \pi)$. $\left(\omega^{2}+f^{2}\right)^{-1}$.
}

matrix whose elements are all zero except for the zeroth element which is $F(t)$, then in the notation of Sec. 2 the equations of motion for coupled "particle and heat path" are

$$
\dot{\mathbf{q}}=\mathbf{p}, \quad \dot{\mathbf{p}}=-\mathbf{A q}+\mathbf{F}(t) .
$$

The formal solution of these equations is

$$
\begin{array}{rl}
\mathbf{q}(t)=\cos \mathbf{A}^{\frac{1}{t}} t & \mathbf{q}(0)+\mathbf{A}^{-\frac{1}{3}} \sin \mathbf{A}^{\frac{1}{2}} t \mathbf{p}(0) \\
& +\int_{0}^{t} d t^{\prime} \frac{\sin \mathbf{A}^{\frac{1}{3}}\left(t-t^{\prime}\right)}{\mathbf{A}^{\frac{1}{t}}} \mathbf{F}\left(t^{\prime}\right), \\
\mathbf{p}(t)=-\mathbf{A}^{\frac{1}{3}} & \sin \mathbf{A}^{\frac{1}{3}} t \mathbf{q}(0)+\cos \mathbf{A}^{\frac{1}{t}} t \mathbf{p}(0) \\
& +\int_{0}^{t} d t^{\prime} \cos \mathbf{A}^{\frac{1}{2}}\left(t-t^{\prime}\right) \mathbf{F}\left(t^{\prime}\right) .
\end{array}
$$

If, now, we take the zeroth element of Eq. (30) for $\dot{\mathbf{p}}$ and substitute (31a) we get

$$
\begin{aligned}
\dot{p}_{0}=- & \sum_{i}\left\|\mathbf{A} \cos \mathbf{A}^{\frac{1}{3}} t\right\|_{0 i} q_{i}(0) \\
& -\sum_{i}\left\|\mathbf{A}^{\frac{1}{3}} \sin \mathbf{A}^{\frac{1}{3}} t\right\|_{0_{i}} p_{i}(0) \\
& -\int_{0}^{t} d t^{\prime}\left\|\mathbf{A}^{\frac{1}{2}} \sin \mathbf{A}^{\frac{1}{3}}\left(t-t^{\prime}\right)\right\|_{0_{0}} F\left(t^{\prime}\right)+F(t) .
\end{aligned}
$$

Next, we eliminate $p_{0}(0)$ between this equation and the zeroth element of Eq. (31b). The result can be written in the form

$$
\begin{aligned}
\dot{p}_{0}-F(t)=-\gamma(t) p_{0}+E(t) & \\
& +\int_{0}^{t} d t^{\prime}\left[\gamma(t)-\gamma\left(t-t^{\prime}\right)\right] \\
& \times\left\|\cos \mathbf{A}^{\frac{1}{3}}\left(t-t^{\prime}\right)\right\|_{00} F\left(t^{\prime}\right),
\end{aligned}
$$

where

$\gamma(t)=\frac{\left\|\mathbf{A}^{\frac{1}{2}} \sin \mathbf{A}^{\frac{1}{t}} t\right\|_{00}}{\left\|\cos \mathbf{A}^{\frac{1}{t}} t\right\|_{00}}=-\frac{d}{d t} \log \left\|\cos \mathbf{A}^{\frac{1}{t}} t\right\|_{00}$,

and

$E(t)=-\sum_{i}\left\{\gamma(t)\left\|\mathbf{A}^{\frac{1}{t}} \sin \mathbf{A}^{\frac{1}{2}} t\right\|_{0 j}\right.$

$\left.+\left\|\mathbf{A} \cos \mathbf{A}^{\frac{1}{2}} t\right\|_{0 i}\right\} q_{i}(0)+\sum_{j}\left\{\gamma(t)\left\|\cos \mathbf{A}^{\frac{1}{2}} t\right\|_{0 i}\right.$

- $\left.\left\|\mathbf{A}^{\frac{1}{2}} \sin \mathbf{A}^{\frac{1}{t}} t\right\|_{0_{i}}\right\} p_{j}(0)$.

Note that the coefficient of $p_{0}(0)$ vanishes in this expression. Equation (32) is the equation of motion for the Brownian particle. The right-hand side is the net force exerted on the Brownian particle by the other particles, i.e., by the heat bath. The first term represents a frictional force with time-dependent "friction coefficient" $\gamma(t)$, the second term represents a fluctuating force $E(t)$ depending upon the initial state of the heat bath, and the third 
term represents a memory effect depending upon the past history of the motion of the Brownian particle.

If we assume that the interaction between the Brownian particle and the heat bath is invariant under translations, we have

$$
\sum_{i} A_{i i}=0
$$

and this in turn implies that (34) may be written

$E(t)=-\sum_{i}^{\prime}\left\{\gamma(t)\left\|\mathbf{A}^{\frac{1}{2}} \sin \mathbf{A}^{\frac{1}{3}} t\right\|_{0 i}\right.$

$\left.+\left\|\mathbf{A} \cos \mathbf{A}^{\frac{1}{2}} t\right\|\right\}\left[q_{j}(0)-q_{0}(0)\right]$

$+\sum_{i}^{\prime}\left\{\gamma(t)\left\|\cos \mathbf{A}^{\frac{1}{2}} t\right\|_{0 i}-\left\|\mathbf{A}^{\frac{1}{2}} \sin \mathbf{A}^{\frac{1}{2}} t\right\|_{0 i}\right\} p_{i}(0)$,

where the prime on the sum denotes the omission of the term $j=0$. Thus the fluctuating force depends only upon the initial coordinates of the particles of the heat bath relative to the initial coordinate of the Brownian particle, and is independent of the initial coordinate and momentum of the Brownian particle.

Consider now what happens when the matrix of interactions is that of the model discussed in Sec. 3, in which the matrix elements are given by (19) with $f(\theta)$ given by (26) in the limit $\omega_{\mathrm{L}} \gg f$. For this model

$$
\left\|\cos \mathbf{A}^{\frac{3}{3}} t\right\|_{00}=e^{-f|\boldsymbol{t}|}
$$

and therefore, from (33) we find that

$$
\lim \gamma(t)=f
$$

which is a constant.

This in turn implies that the last term on the right-hand side of (32) (the memory-effect term) becomes, in the limit, identically 0 !

With these results, (32) takes the form

$$
\dot{p}_{0}-F(t)=-f p_{0}+E(t),
$$

with

$$
\begin{aligned}
E(t)= & -\sum_{j}\left\|f \mathbf{A}^{\frac{1}{2}} \sin \mathbf{A}^{\frac{1}{3}} t+\mathbf{A} \cos \mathbf{A}^{\frac{1}{t}} t\right\|_{0 i} q_{i}(0) \\
& +\sum_{j}\left\|f \cos \mathbf{A}^{\frac{1}{2}} t-\mathbf{A}^{\frac{1}{2}} \sin \mathbf{A}^{\frac{1}{2}} t\right\|_{0 i} p_{j}(0) .
\end{aligned}
$$

Equation (39) is the Langevin equation.

It remains to prove that the statistical properties of $E(t)$ become (again in the limit $N \rightarrow \infty, \omega_{\mathrm{L}} \gg f$ ) those of a purely random Gaussian process. This depends, of course on the statistical assumptions concerning initial positions and momenta.

We would like to require that at $t=0$ the heat bath is in equilibrium at temperature $T$ and the simplest way of doing this is to assume that the initial distribution is the canonical distribution (6). This however is, strictly speaking, impossible since
(35) implies that $\omega_{0}=0$ so that $\operatorname{det} \mathbf{A}=0$ and the canonical distribution becomes improper. The difficulty is not serious and can be remedied, e.g., by slightly modifying the matrix $\mathbf{A}$; i.e., replacing it by (I is the unit matrix)

$$
\mathbf{A}+\varepsilon_{N} \mathbf{I},
$$

where $\varepsilon_{N}$, though positive for every finite $N$, approaches 0 as $N \rightarrow \infty$ (as fast as one pleases). Now (35) is only approximately true and the canonical distribution (6) is proper. We hope that the reader does not become unduly confused by our use of the symbol $\mathbf{A}$ to denote three different matrices. We use it to denote the finite cyclic matrix (12), the modification (41), and last but not least for the infinite cyclic matrix (defined only with the cutoff $\omega_{L}$ ). Clearly, since the distribution (6) of the $q_{j}(0)$ and $p_{j}(0)$ is Gaussian, $E(t)$ is a Gaussian process. We can form its covariance with the help of the results of Appendix 1, and, in the limit considered throughout this paper, we find that

$\left\langle E(t) E\left(t^{\prime}\right)\right\rangle=k T\left\|\left(f^{2}+\mathbf{A}\right) \cos \mathbf{A}^{\frac{t}{3}}\left(t-t^{\prime}\right)\right\|_{00}$.

But the matrix $\mathbf{A}$ is given by the model of Sec. 3; hence, using (21), we find

$$
\begin{aligned}
\langle E(t) & \left.E\left(t^{\prime}\right)\right\rangle \\
= & \frac{k T}{2 \pi} \int_{-\pi}^{\pi} d \theta\left(f^{2}+f^{2} \tan ^{2} \frac{\theta}{2}\right) \cos f \tan \frac{\theta}{2}\left(t-t^{\prime}\right) \\
= & \frac{k T f}{\pi} \int_{-\infty}^{\infty} d \omega \cos \omega\left(t-t^{\prime}\right) .
\end{aligned}
$$

This last integral is the well known expression for the Dirac delta function, so we have

$$
\left\langle E(t) E\left(t^{\prime}\right)\right\rangle=2 f k T \delta\left(t-t^{\prime}\right) .
$$

Thus $E(t)$ is a purely random, Gaussian, stochastic process and Eq. (39) is the Langevin equation for Brownian motion.

In order that the equation of motion (32) become the Langevin equation it is necessary that (i) the friction constant $\gamma(t)$ be independent of time; (ii) the stochastic process $E(t)$ be a purely random Gaussian process; (iii) the memory effects disappear.

We feel that it is striking that, for our model, these three properties are intimately related. Undoubtedly this fact is of much more general significance.

\section{THE MOTION OF COUPLED QUANTUM OSCILLATORS}

We turn now to the question of the changes required for a quantum mechanical description of 
the motion of coupled oscillators. The answer is that much of our previous discussion is, formally at any rate, entirely unchanged. Thus in our discussion of Sec. 2, the Hamiltonian (2) is unchanged, but the coordinates $q_{i}$ and momenta $p_{i}$ are now operators whose commutation rules are

$$
\begin{gathered}
{\left[q_{i}, q_{k}\right]=\left[p_{i}, p_{k}\right]=0,} \\
{\left[q_{i}, p_{k}\right]=i \hbar \delta_{j k} .}
\end{gathered}
$$

The equations of motion (3) are the equations of motion in the Heisenberg picture, and their solution (4) relates the Heisenberg operators at time $t$ to the operators at the initial time.

At $t=0$, we assume the system is in equilibrium at temperature $T$. In the quantum description this means that the initial state of the system is described by the density matrix corresponding to the canonical ensemble:

$$
\rho(\mathbf{q}(0), \mathbf{p}(0))=\exp \{-\beta H(\mathbf{q}(0), \mathbf{p}(0))\} .
$$

The expectation of any function $F(\mathrm{q}(0), \mathrm{p}(0))$ of the operators $q(0), p(0)$ given by

$$
\left\langle F^{\prime}\right\rangle=\frac{\operatorname{Tr}\{F(\mathbf{q}(0), \mathbf{p}(0)) \rho(\mathbf{q}(0), \mathbf{p}(0))\}}{\operatorname{Tr}\{\rho(\mathbf{q}(0), \mathbf{p}(0))\}} .
$$

Just as in the classical case, we now consider the properties of the stochastic operators $q_{i}(t)$ and $p_{i}(t)$ which result from the equation of motion and the initial density matrix. These properties we describe in terms of the correlation functions, the simplest being the pair correlation functions. These are obtained in Appendix 2; the results are

$$
\begin{aligned}
& \left\langle p_{i}(t) p_{k}(t+\tau)\right\rangle \\
& =\left\|\frac{\hbar \mathbf{A}^{\frac{1}{3}}}{2}\left[\operatorname{coth} \frac{\hbar \mathbf{A}^{\frac{1}{2}}}{2 k T} \cos \mathbf{A}^{\frac{1}{2}} \tau+i \sin \mathbf{A}^{\frac{1}{3}} \tau\right]\right\|_{i k} \\
& \left\langle q_{i}(t) q_{k}(t+\tau)\right\rangle \\
& =\left\|\frac{\hbar}{2 \mathbf{A}^{\frac{1}{2}}}\left[\operatorname{coth} \frac{\hbar \mathbf{A}^{\frac{1}{2}}}{2 k T} \cos \mathbf{A}^{\frac{1}{2}} \tau+i \sin \mathbf{A}^{\frac{1}{\tau}}\right]\right\|_{i k} \\
& \left\langle q_{i}(t) p_{k}(t+\tau)\right\rangle \\
& =\left\|\frac{\hbar}{2}\left[-\operatorname{coth} \frac{\hbar \mathbf{A}^{\frac{1}{2}}}{2 k T} \sin \mathbf{A}^{\frac{1}{2}} \tau+i \cos \mathbf{A}^{\frac{1}{2}} \tau\right]\right\|_{i k} .
\end{aligned}
$$

Note that in the limit $\hbar=0$, these expressions become identical with the classical expressions (9). As indicated in Appendix 2, the higher correlations are given by the rule:

Correlations of an odd number of $q$ 's and $p$ 's vanish. The correlation of an even number of $q$ 's and $p$ 's is equal to the sum of products of pair cor- relations, the sum being over all possible pairings of the operators, with order preserved.

Except for the italicized proviso that the order be preserved, this rule is identical with the classical rule for a Gaussian random process. ${ }^{8}$ The stochastic operators also have the classical stationarity property; the correlations depend only upon the time differences. There are, however, obvious differences between the properties of the stochastic operators and the properties of the corresponding classical stochastic process. The principal differences arise from the fact that the quantum operators do not commute. Thus there are many (indeed, an infinity!) of correlations of the quantum operators which can be associated with a given classical correlation, corresponding to different orderings of the operators. As a simple example,

$$
\langle\epsilon q p+(1-\epsilon) p q\rangle_{q u a n t u m} \rightarrow\langle q p\rangle_{\text {clssaical }},
$$

where $\epsilon$ is an arbitrary complex number. Another difficulty comes from the fact that the product of two noncommuting Hermitian operators is not Hermitian, and, therefore, does not correspond to a physical observable. We see this difficulty explicitly in the correlations (47), which are complex functions, whereas the expectation value of a physical observable should be real.

The difficulties we mention would be largely resolved if we had a conventional definition of the product of operators with the following properties:

(a) The product is independent of the order of the operators.

(b) The product of a number of Hermitian operators is itself Hermitian.

(c) The classical pair decomposition rule for expressing higher correlations in terms of pair correlations holds.

For our system of coupled ascillators there is such a conventional product, namely the ordered product or normal product introduced in quantum field theory"; it is defined as follows. In Appendix 2 we show how the operators $q(0)$ and $p(0)$ can be expanded in terms of creation and annihilation operators for the normal modes;

$$
\begin{aligned}
& \mathbf{q}(0)=i \sum_{s} \xi^{(s)}\left(\frac{\hbar}{2 \omega_{s}}\right)^{\frac{1}{2}}\left(a_{s}-a_{s}^{*}\right), \\
& \mathbf{p}(0)=\sum_{s} \xi^{(s)}\left(\frac{\hbar \omega_{s}}{2}\right)^{\frac{*}{2}}\left(a_{s}+a_{s}^{*}\right) .
\end{aligned}
$$

See, e.g., Wang and Uhlenbeck, Ref. 2.

'See, e.g., G.-C. Wick, Phys. Rev, 80, 268 (1950). 
Here $\xi^{(s)}$ is the eigenvector of the interaction matrix $A$, and $\omega_{s}^{2}$ is the associated eigenvalue

$$
\mathbf{A} \xi^{(s)}=\omega_{s}^{2} \xi^{(s)} \text {. }
$$

The operator $a_{s}$ is the annihilation operator for the sth normal mode and $a_{\mathrm{s}}^{*}$ is the corresponding creation operator. Their commutation rules are

$$
\left[a_{s}, a_{r}^{*}\right]=\delta_{s r}, \quad\left[a_{s}, a_{r}\right]=\left[a_{s}^{*}, a_{r}^{*}\right]=0 .
$$

The time-dependent operators are expressed in terms of the $a_{s}$ and $a_{s}^{*}$ by inserting (48) in (4). We find

$$
\begin{aligned}
& \mathbf{q}(t)=i \sum_{s} \xi^{(s)}\left(\frac{\hbar}{2 \omega_{s}}\right)^{\frac{1}{2}}\left(a_{s} e^{-i \omega_{\bullet} t}-a_{s}^{*} e^{i \omega \cdot t}\right), \\
& \mathbf{p}(t)=\sum_{s} \xi^{(s)}\left(\frac{\hbar \omega_{s}}{2}\right)^{\frac{1}{2}}\left(a_{s} e^{-i \omega_{\diamond} t}+a_{s}^{*} e^{i \omega_{*} t}\right) .
\end{aligned}
$$

The normal product of a number of the operators $a_{a}$ and $a^{*}$ is defined to be that product in which all the $a_{s}^{*}$ are written to the left of all the $a_{s}$. Because of the commutation rules (50), this defines a unique order. The normal product of a number of operators $q_{i}(t)$ and $p_{i}(t)$ is the product in which the expansions (51) are used and each product of the $a_{s}$ and $a_{s}^{*}$ is written in normal form. We denote the normal product by a colon placed before and after the product of factors. As an example,

$$
\begin{aligned}
: q_{j}\left(t_{1}\right) p_{k}\left(t_{2}\right): \equiv & i \frac{\hbar}{2} \sum_{s} \sum_{r}\left(\frac{\omega_{r}}{\omega_{s}}\right)^{j} \xi_{j}^{(s)} \xi_{k}^{(r)}\left[a_{s} a_{r} e^{-i\left(\omega_{\theta} t_{1}+\omega_{r} t_{2}\right)}\right. \\
& -a_{a}^{*} a_{r} e^{i\left(\omega_{0} t_{2}-\omega_{r} t_{s}\right)}+a_{r}^{*} a_{s} e^{-i\left(\omega_{8} t_{2}-\omega_{r} t_{g}\right)} \\
& \left.-a_{s}^{*} a_{r}^{*} e^{i\left(\omega_{s} t_{1}+\omega_{r} t_{2}\right)}\right] .
\end{aligned}
$$

The normal product fulfills our requirements. It us clearly independent of the order of the factors, and, since $a_{s}^{*}$ is the Hermitian conjugate of $a_{0}$, the normal product of a number of Hermitian operators is Hermitian. Using the results given in Appendix 2, we can show that the pair correlations of normal products of the $q_{i}(t)$ and $p_{k}(t)$ are

$$
\begin{aligned}
& \left\langle: p_{i}(t) p_{k}(t+\tau):\right\rangle=\left\|P\left(\hbar \mathbf{A}^{\frac{1}{2}} / k T\right) \cos \mathbf{A}^{\frac{1}{\tau}} \tau\right\|_{i k}, \\
& \left\langle: q_{i}(t) q_{k}(t+\tau):\right\rangle=\left\|P\left(\hbar \mathbf{A}^{\frac{1}{2}} / k T\right) \mathbf{A}^{-1} \cos \mathbf{A}^{\frac{1}{2}} \tau\right\|_{i k}, \\
& \left\langle: q_{i}(t) p_{k}(t+\tau):\right\rangle=-\left\|P\left(\hbar \mathbf{A}^{\frac{1}{2}} / k T\right) \mathbf{A}^{-\frac{1}{2}} \sin \mathbf{A}^{\frac{1}{2}} \tau\right\|_{i k} .
\end{aligned}
$$

Here we have introduced the Planck function

$$
P(x) \equiv k T x /\left(e^{x}-1\right) .
$$

When $x=\hbar \omega / k T$ the Planck function is the average energy, relative to the ground state, of a quantum oscillator of frequency $\omega$. As $x \rightarrow 0, P(x) \rightarrow k T$, the classical equipartition energy.

The higher correlations of normal products are given by the well-known rule for a Gaussian random process:

The correlation of the normal product of an odd number of $q$ 's and $p$ 's vanishes. The correlation of the normal product of an even number of $q$ 's and $p$ 's is equal to the sum of products of pair correlation of normal products, the sum being over all possible pairings.

What we can say, then, is that the correlations of normal products of our stochastic operators are identical with those of a stationary Gaussian process whose pair correlations are given by $(52) .^{10}$

Finally, if we fix our attention on a single oscillator, the one with index 0 , the momentum autocorrelation is

$\left\langle p_{0}(t) p_{0}(t+\tau):\right\rangle=\left\|P\left(\hbar \mathbf{A}^{\frac{1}{2}} / k T\right) \cos \mathbf{A}^{\frac{1}{2}} \tau\right\|_{00}$.

Just as in the corresponding classical process, we can ask whether there is an interaction matrix $\mathbf{A}$ for which (54) is an exponential and, therefore, the corresponding Gaussian process is Markoffian. The answer is that we can, but that it is temperaturedependent, and, therefore, not of physical interest. If we use the model discussed in Sec. 3 , in which the matrix elements are given by (19) with $f(\theta)$ given by (26) in the limit $\omega_{L} \gg f$, we find (54) becomes

$$
\begin{aligned}
\left\langle: p_{0}(t) p_{0}(t\right. & +\tau):\rangle \\
& =\frac{2}{\pi} \int_{0}^{\infty} d \omega P\left(\frac{\hbar \omega}{k T}\right) \frac{f}{\omega^{2}+f^{2}} \cos \omega \tau .
\end{aligned}
$$

In the limit $\hbar \rightarrow 0$, this becomes identical with the classical result obtained earlier, and the process becomes Markoffian.

\section{THE QUANTUM LANGEVIN EQUATION}

The formal manipulations we used in Sec. 4 to derive the Langevin equation are unchanged when we interpret the $q$ 's and $p$ 's as quantum operators. In particular, when the matrix of interactions is that given by the model of Sec. 3 , in which the matrix elements are given by (19) with $f(\theta)$ given by (26) in the limit $\omega_{\mathrm{L}} \gg f$, we obtain an operator equation of motion which is formally identical with Langevin equation (39). That is

$$
\dot{p}_{0}-F(t)=-f p_{0}+E(t),
$$

where

$$
\begin{aligned}
E(t)= & -\sum_{j}\left\|f \mathbf{A}^{\frac{1}{2}} \sin \mathbf{A}^{\frac{1}{t}} t+\mathbf{A} \cos \mathbf{A}^{\frac{1}{2}} t\right\|_{0_{j}} q_{j}(0) \\
& +\sum_{i}\left\|f \cos \mathbf{A}^{\frac{1}{3}} t-\mathbf{A}^{\frac{1}{2}} \sin \mathbf{A}^{\frac{1}{3}} t\right\|_{0 i} p_{i}(0)
\end{aligned}
$$

${ }_{10}$ Essentially the same point is made in connection with the quantum description of statistical light beams by $\mathrm{E}$. C. G. Sudarshan, Phys. Rev. Letters 10, 277 (1963). See also R. J. Glauber, Phys. Rev. Letters 10, 84 (1963). 
The operator Langevin equation is an equation of motion for the time-dependent Heisenberg operators $p_{0}(t)$ and $q_{0}(t)$. The operator $F(t)$ is the external force operator,

$$
F(t) \equiv(i \hbar)^{-1}\left[p_{0}(t), V\left(q_{0}(t), t\right)\right],
$$

with $V\left(q_{0}, t\right)$ the (time-dependent) potential of the external force. The random-force operator $E(t)$ is in fact independent of the operators $p_{0}(0)$ and $q_{0}(0)$ since their coefficients in the expression $\left(40^{\prime}\right)$ vanish for our model of the interaction matrix. Because of the commutation rules (44), this means that

$$
\left[q_{0}(0), E(t)\right]=\left[p_{0}(0), E(t)\right]=0 .
$$

We assume that the statistical state of the initial coordinates and momenta of the heat bath is described by the density matrix (45). Just as in the classical case, this means that at $t=0$ the heat bath (i.e., the oscillators other than the Brownian particle) is in equilibrium with a fictitious force-free Brownian particle. Since the random-force operator $E(t)$ is independent of the initial coordinate and momentum of the Brownian particle, its stochastic properties are unaffected by the dependence of the density matrix upon these operators. The covariance of the normal product is readily obtained using the results (52), we find

$$
\begin{aligned}
\langle: E(t) E(t & +\tau):\rangle \\
& =\left\|\left(f^{2}+\mathbf{A}\right) P\left(\hbar \mathbf{A}^{\frac{1}{2}} / k T\right) \cos \mathbf{A}^{\frac{1}{2}} \tau\right\|_{00} .
\end{aligned}
$$

But for our model, we may use the general expression (21), with $f(\theta)$ given by (25). Hence

$$
\begin{aligned}
\langle: E(t) E(t+\tau) & :\rangle=\frac{1}{2 \pi} \int_{-\pi}^{\pi} d \theta f^{2}\left(1+\tan ^{2} \frac{1}{2} \theta\right) \\
& \times P\left(\frac{\hbar f}{k T}\left|\tan \frac{1}{2} \theta\right|\right) \cos \left(f \tau \tan \frac{1}{2} \theta\right) \\
& =\frac{2 f}{\pi} \int_{0}^{\infty} d \omega P\left(\frac{\hbar \omega}{l T}\right) \cos \omega \tau .
\end{aligned}
$$

Correlations of higher normal products of $E(t)$ are again given by the rule for a Gaussian random process. Hence, the stochastic properties of the random operator $E(t)$, as expressed by the correlations of normal products, are identical with those of a stationary Gaussian process whose covariance is given by (59). In the limit $\hbar \rightarrow 0$, this covariance approaches the corresponding classical covariance (43), which is the covariance of a purely random Gaussian process. However, for finite $\hbar,(59)$ is the covariance of a Gaussian process which is not even Markoffian. This is the chief difference between the quantum and classical Langevin equations for our model.

As an elementary application, consider the motion in a constant field of force,

$$
F(t)=\mathcal{E} \text {. }
$$

The solution of the operator Langevin equation is

$$
\begin{aligned}
& p_{0}(t)=e^{-f t} p_{0}(0)+f^{-1} \mathcal{E}\left(1-e^{-f t}\right) \\
& +\int_{0}^{t} d t^{\prime} e^{-f\left(t-t^{\prime}\right)} E\left(t^{\prime}\right) .
\end{aligned}
$$

If we average this expression over the initial state of the heat bath, we find ${ }^{11}$

$$
\left\langle p_{0}(t)\right\rangle=e^{-f t} p_{0}(0)+f^{-1} \mathcal{E}\left(1-e^{-f t}\right),
$$

since $\langle E(t)\rangle=0$. After a long time $\left(t \gg f^{-1}\right)$ we find

$$
\left\langle p_{0}(t)\right\rangle \sim f^{-1} \varepsilon \text {. }
$$

This is the analog for our model of Ohm's law. The left-hand side is the "current," which in the steady state is proportional to the applied field, and inversely proportional to the "resistance," i.e., the friction constant.

The mean-square "fluctuation current" may also be obtained from (60)

$\left\langle:\left[p_{0}(t)-\left\langle p_{0}(t)\right\rangle\right]^{2}:\right\rangle$

$=\int_{0}^{t} d t^{\prime} e^{-f\left(t-t^{\prime}\right)} \int_{0}^{t} d t^{\prime \prime} e^{-f\left(t-t^{\prime \prime}\right)}\left\langle: E\left(t^{\prime}\right) E\left(t^{\prime \prime}\right):\right\rangle$

Note that we have used the normal product in the definition of the squared fluctuation. Using the expression (58) for the covariance of $E(t)$, we find

$$
\begin{aligned}
\left\langle:\left[p_{0}(t)\right.\right. & \left.\left.-\left\langle p_{0}(t)\right\rangle\right]^{2}:\right\rangle \\
& =\frac{2 f}{\pi} \int_{0}^{\infty} d \omega P\left(\frac{\hbar \omega}{k T}\right)\left|\frac{1-e^{-(f-i \omega) t}}{f-i \omega}\right|^{2},
\end{aligned}
$$

where we have used the elementary result

$$
\begin{gathered}
\int_{0}^{t} d t^{\prime} \int_{0}^{t} d t^{\prime \prime} e^{-f\left(t-t^{\prime}\right)} e^{-f\left(t-t^{\prime \prime}\right)} \cos \omega\left(t^{\prime}-t^{\prime \prime}\right) \\
=\left|\left(1-e^{-(f-i \omega) t}\right) /(f-i \omega)\right|^{2}
\end{gathered}
$$

In the steady state, i.e., when $t \gg f^{-1},(65)$ becomes

$$
\left\langle:\left[p_{0}(t)-\left\langle p_{0}(t)\right\rangle\right]^{2}:\right\rangle=\frac{2 f}{\pi} \int_{0}^{\infty} d \omega P\left(\frac{\hbar \omega}{k T}\right) \frac{1}{\omega^{2}+f^{2}} .
$$

This is the analog for our model of the Nyquist formula, which relates the noise power spectrum

\footnotetext{
II The result of the average over the states of the heat bath is still in general an operator function of $q_{0}(0)$ and $p_{0}(0)$. We trust that our definition of this average, which involves an average over the initial coordinate and momentum of a fictitious Brownian particle, is not confusing.
} 
to the resistance and the absolute temperature. ${ }^{12}$ Note that if we had used the ordinary product instead of the normal product, we would find

$$
\begin{aligned}
& \left\langle\left[p_{0}(t)-\left\langle p_{0}(t)\right\rangle\right]^{2}\right\rangle \\
& \equiv\left\langle\left[p_{0}(t)-\left\langle p_{0}(t)\right\rangle\right] \cdot\left[p_{0}(t)-\left\langle p_{0}(t)\right\rangle\right]\right\rangle \\
& =\left\langle:\left[p_{0}(t)-\left\langle p_{0}(t)\right\rangle\right]^{2}:\right\rangle+\frac{2 f}{\pi} \int_{0}^{\omega L} d \omega \frac{\hbar \omega}{2} \frac{1}{\omega^{2}+f^{2}} .
\end{aligned}
$$

The added integral is clearly related to the zeropoint fluctuations of the heat bath. It is also divergent in the limit $\omega_{L} \rightarrow \infty$, so we have retained this cutoff explicitly. Which of the possible definitions of the product corresponds to an experimental measurement of the fluctuation spectrum? This, in the last analysis, must be determined by the experiment itself. We tend to the opinion that the normal product is the physically appropriate definition, since it leads to a noise spectrum which vanishes at absolute zero.

\section{BROWNIAN MOTION OF A QUANTUM OSCILLATOR}

As a second application of the quantum Langevin equation, we consider the case of the harmonic oscillator, for which the external force is ${ }^{13}$

$$
F(t)=-\kappa^{2} q_{0}(t),
$$

where $\kappa$ is the natural frequency of the oscillator. The quantum Langevin equation $\left(39^{\prime}\right)$ becomes

$$
\dot{p}_{0}+\kappa^{2} q_{0}=-f p_{0}+E(t) \text {, }
$$

to which we must append the equation

$$
\dot{q}_{0}=p_{0} \text {. }
$$

The solution of this pair of coupled equations is

$$
\begin{aligned}
q_{0}(t)= & e^{-\frac{1}{2} f t}\left\{[\cos \nu t+(f / 2 \nu) \sin \nu t] q_{0}(0)\right. \\
& \left.+\nu^{-1} \sin \nu t p_{0}(0)\right\} \\
& +\int_{0}^{t} d t^{\prime} e^{-\frac{1}{2} f\left(t-t^{\prime}\right)} \frac{1}{\nu} \sin \nu\left(t-t^{\prime}\right) E\left(t^{\prime}\right), \\
p_{0}(t)=e^{-\frac{1}{2} / t}\{- & \left(\kappa^{2} / \nu\right) \sin \nu t q_{0}(0) \\
& \left.+[\cos \nu t-(f / 2 \nu) \sin \nu t] p_{0}(0)\right\} \\
& +\int_{0}^{t} d t^{\prime} e^{-\frac{1}{2}\left(t-t^{\prime}\right)}\left[\cos \nu\left(t-t^{\prime}\right)\right. \\
& \left.-(f / 2 \nu) \sin \nu\left(t-t^{\prime}\right)\right] E\left(t^{\prime}\right) .
\end{aligned}
$$

${ }^{12}$ For elementary discussion of the Nyquist formula and its quantum generalization see C. Kittel, Elementary Statistical Physics (John Wiley \& Sons, Inc., New York, 1958), pp. 141-153. See also J. Lawson and G. E. Uhlenbeck, Threshold Noise Signals (McGraw-Hill Book Company, Inc., New York, 1950), especially pp. 64-79.

${ }_{13}$ The Brownian motion of a quantum oscillator is considered in a paper by J. Schwinger, J. Math. Phys. 2, 407 (1961).
Here

$$
\nu=\left(\kappa^{2}-\frac{1}{4} f^{2}\right)^{\frac{1}{2}},
$$

and we restrict our discussion to the underdamped case, where $\kappa>\frac{1}{2} f$, so $\nu$ is real and positive.

The mean motion of the oscillator is described by the operators obtained by averaging (72) and (73) over the initial states of the heat bath. Since $\langle E(t)\rangle=$ 0 , we have

$$
\begin{gathered}
\left\langle q_{0}(t)\right\rangle=e^{-\frac{1}{2} / t}\left\{[\cos \nu t+(f / 2 \nu) \sin \nu t] q_{0}(0)\right. \\
\left.+\nu^{-1} \sin \nu t p_{0}(0)\right\}, \\
\left\langle p_{0}(t)\right\rangle=e^{-\frac{1}{2} / t}\left\{-\kappa^{2} \nu^{-1} \sin \nu t q_{0}(0)\right. \\
\left.+[\cos \nu t-(f / 2 \nu) \sin \nu t] p_{0}(0)\right\} .
\end{gathered}
$$

These are just the operator solutions of the average of the equations of motion (70) and (71). We see from (74) that these operators vanish for $t \gg f^{-1}$; the mean motion of the oscillator vanishes for times long compared with the "macroscopic relaxation time" $f^{-1}$.

To see more precisely what we mean by the operators (74), consider the coordinate representation. The Heisenberg state of the oscillator is then specified by a time-independent wavefunction $\psi\left[q_{0}(0)\right]$, and the initial momentum operator is represented by $-i \hbar \partial / \partial q_{0}(0)$. The simplest kind of question we can ask about the Heisenberg operators is their expectation value, denoted by a subscript "ex" to the operator. For example,

$$
\begin{aligned}
&\left\langle q_{0}(t)\right\rangle_{\mathrm{ex}} \equiv \int_{-\infty}^{\infty} d q_{0}(0) \psi^{*}\left[q_{0}(0)\right]\left\langle q_{0}(t)\right\rangle \psi\left[q_{0}(0)\right] \\
&=e^{-\frac{1}{2} f t}\{(\cos \nu t+(f / 2 \nu) \sin \nu t)\left[q_{0}(0)\right]_{\mathrm{ex}} \\
&\left.+\nu^{-1} \sin \nu t\left[p_{0}(0)\right]_{\mathrm{ex}}\right\} .
\end{aligned}
$$

As a simple illustration, if the wavefunction is

$$
\psi\left[q_{0}(0)\right]=(\alpha / \pi)^{\frac{1}{2}} e^{-\frac{1}{2} \alpha\left(q_{0}(0)-x_{0}\right)^{*}},
$$

then

$$
\left[q_{0}(0)\right]_{\mathrm{ex}}=x_{0}, \quad\left[p_{0}(0)\right]_{\mathrm{ex}}=0,
$$

and

$$
\begin{aligned}
& \left\langle q_{0}(t)\right\rangle_{\text {ex }}=e^{-\frac{1}{2} f t}[\cos \nu t+(f / 2 \nu) \sin \nu t] x_{0}, \\
& \left\langle p_{0}(t)\right\rangle_{\text {ex }}=-e^{-\frac{1}{2} f t \kappa^{2} \nu^{-1}} \sin \nu t x_{0} .
\end{aligned}
$$

The operator describing the fluctuation of the displacement of the oscillator about the mean displacement is

$$
\begin{aligned}
q_{0}(t)- & \left\langle q_{0}(t)\right\rangle \\
& =\int_{0}^{t} d t^{\prime} e^{\left.-\xi(t)-t^{\prime}\right)} \frac{\sin \nu\left(t-t^{\prime}\right)}{\nu} E\left(t^{\prime}\right) .
\end{aligned}
$$


The mean-square fluctuation in displacement we express as the mean of the square of (79), written as a normal product. Using (59), we find

$$
\begin{aligned}
& \left\langle:\left[q_{0}(t)-\left\langle q_{0}(t)\right\rangle\right]^{2}:\right\rangle=\frac{2 f}{\pi} \int_{0}^{\infty} d \omega P\left(\frac{\hbar \omega}{k T}\right) \\
& \times\left|\frac{1-e^{-\left(\frac{3}{2} f-i \omega\right) t}\left[\cos \nu t+\left(\frac{1}{2} f-i \omega\right) \nu^{-1} \sin \nu t\right]}{\omega^{2}-\kappa^{2}+i f \omega}\right|^{2} .
\end{aligned}
$$

Here we have used the following result of an elementary but tedious integration

$$
\begin{aligned}
& \int_{0}^{t} d t^{\prime} \int_{0}^{t} d t^{\prime \prime} e^{-\frac{t}{2} \int\left(t-t^{\prime}\right)} \frac{\sin \nu\left(t-t^{\prime}\right)}{\nu} \\
& \quad \times e^{-\frac{1}{2} \int\left(t-t^{\prime \prime}\right)} \frac{\sin \nu\left(t-t^{\prime \prime}\right)}{\nu} \cos \omega\left(t^{\prime}-t^{\prime \prime}\right) \\
& =\left|\frac{\left.1-e^{-\left(\frac{1}{2} f-i \omega\right) t}\left[\cos \nu t+\left(\frac{1}{2} f-i \omega\right)\right)^{-1} \sin \nu t\right]}{\omega^{2}-\kappa^{2}+i f \omega}\right|^{2} .
\end{aligned}
$$

The time dependence of the mean fluctuation in displacement, as expressed by (80), is rather complicated. It does, however, have the simple feature that, for times long compared with the relaxation time $f^{-1}$, it approaches an equilibrium value

$$
\begin{aligned}
& \left\langle:\left[q_{0}(t)-\left\langle q_{0}(t)\right\rangle\right]^{2}:\right\rangle_{\text {eq }} \\
& \quad=\frac{2 f}{\pi} \int_{0}^{\infty} d \omega \frac{P(\hbar \omega / k T)}{\left(\omega^{2}-\kappa^{2}\right)^{2}+\omega^{2} f^{2}} .
\end{aligned}
$$

In the classical limit we use the property of the Planck function (53)

$$
P(\hbar \omega / k T) \rightarrow k T, \quad \text { as } \hbar \rightarrow 0 .
$$

The remaining integral in (82) is elementary:

$$
\frac{2 f}{\pi} \int_{0}^{\infty} d \omega \frac{1}{\left(\omega^{2}-\kappa^{2}\right)^{2}+\omega^{2} f^{2}}=\kappa^{-2}
$$

and we find

$$
\left\langle:\left[q_{0}(t)-\left\langle q_{0}(t)\right\rangle\right]^{2}:\right\rangle_{\text {eq }} \rightarrow k T / \kappa^{2}, \quad \text { as } \hbar \rightarrow 0,
$$

which is the classical equipartition result.

Another simple limit of (82) is the weak-coupling limit, in which the coupling of the Brownian particle to the heat bath is weak compared with the oscillator coupling. That is, $\kappa \gg f$. In this limit the resonance denominator in (82) becomes sharply peaked at $\omega=\kappa$, with a width $\approx f$. Hence, we can evaluate the Planck function at $\omega=\kappa$ and perform the remaining integral using (84). We find

$$
\left\langle:\left[q_{0}(t)-\left\langle q_{0}(t)\right\rangle\right]^{2}:\right\rangle_{\mathrm{eq}} \rightarrow \frac{1}{\kappa} P\left(\frac{\hbar_{\kappa}}{k T}\right), \text { for } \kappa \gg f \text {. }
$$

This is the well-known Planck result for the mean- square displacement of an independent oscillator of frequency $\kappa$ at temperature $T$.

\section{APPENDIX 1: CORRELATIONS II A SYSTEM OF COUPLED CLASSICAL OSCILLATORS}

Consider first the correlations of the initial values of the coordinates and momenta, whose distribution is [c.f. Eq. (6)]

$$
\begin{aligned}
& D(\mathbf{q}(0), \mathrm{p}(0))=(2 \pi / \beta)^{2 N+1}(\operatorname{det} \mathbf{A})^{-\frac{1}{2}} \\
& \quad \times \exp \left\{-\frac{\beta}{2}\left[\sum_{i} p_{i}^{2}(0)+\sum_{j, k} q_{i}(0) A_{j k} q_{k}(0)\right]\right\} .
\end{aligned}
$$

Since this is a Gaussian distribution, all higher correlations can be expressed in terms of the pair correlations. These are $(\beta=1 / k T)$ :

$$
\begin{aligned}
& \left\langle p_{j}(0) p_{k}(0)\right\rangle=k T \delta_{j k}, \\
& \left\langle p_{j}(0) q_{k}(0)\right\rangle=0, \\
& \left\langle q_{j}(0) q_{k}(0)\right\rangle=k T\left\|\mathbf{A}^{-1}\right\|_{j k} .
\end{aligned}
$$

These expressions are consequences of well-known integral formulas for Gaussian distributions. ${ }^{14}$

The pair correlations for the time-dependent coordinates and momenta are found from (4), using (2). We have for the momentum correlation:

$$
\begin{aligned}
\left\langle p_{i}(t) p_{k}(t+\tau)\right\rangle=\sum_{m, n}\left\{\left\|\mathbf{A}^{\frac{1}{3}} \sin \mathbf{A}^{\frac{1}{2}} t\right\|_{i m}\right. \\
\quad \times\left\|\mathbf{A}^{\frac{1}{3}} \sin \mathbf{A}^{\frac{1}{2}}(t+\tau)\right\| \|_{k n}\left\langle q_{m}(0) q_{n}(0)\right\rangle \\
\left.\quad+\left\|\cos \mathbf{A}^{\frac{1}{2}} t\right\|_{i m}\left\|\cos \mathbf{A}^{\frac{1}{3}}(t+\tau)\right\| \|_{k_{n}}\left(p_{m}(0) p_{n}(0)\right\rangle\right\} \\
=k T\left\{\| \sin \mathbf{A}^{\frac{1}{2}} t \sin \mathbf{A}^{\frac{3}{3}}(t+\tau)\right. \\
\quad+\cos \mathbf{A}^{\frac{1}{1}} t \cos \mathbf{A}^{\frac{1}{2}}(t+\tau) \|_{i k},
\end{aligned}
$$

where we have used the fact that the matrix $A$ is symmetric. Using the formula for the cosine of the difference of two angles, we find

$$
\left\langle p_{i}(t) p_{k}(t+\tau)\right\rangle=k T\left\|\cos \mathbf{A}^{\frac{1}{2}} \tau\right\|_{j k} .
$$

In a similar way, we can also show

$$
\begin{aligned}
& \left\langle q_{j}(t) p_{k}(t+\tau)\right\rangle=-k T\left\|\mathbf{A}^{-\frac{1}{2}} \sin \mathbf{A}^{\frac{1}{2}} \tau\right\|_{i k,} \\
& \left\langle q_{i}(t) q_{k}(t+\tau)\right\rangle=k T\left\|\mathbf{A}^{-1} \cos \mathbf{A}^{\frac{1}{2}} \tau\right\|_{i k} .
\end{aligned}
$$

Since the process is Gaussian, the higher correlations are given by the following rule ${ }^{8}$ :

The correlation of an odd number of coordinates and momenta vanish. The correlation of an even number of coordinates and momenta is equal to the

${ }^{14}$ See, e.g., H. Cramer, Mathematical Methods of Statistics (Princeton University Press, Princeton, New Jersey, 1945), p. 118 . 
sum of products of pair correlations, the sum being over all pairings.

For example,

$$
\begin{aligned}
\left\langle q_{i}\left(t_{1}\right) q_{k}\left(t_{2}\right) q_{l}\left(t_{3}\right) p_{m}\left(t_{4}\right)\right\rangle & =\left\langle q_{j}\left(t_{1}\right) q_{k}\left(t_{2}\right)\right\rangle\left\langle q_{l}\left(t_{3}\right) p_{m}\left(t_{4}\right)\right\rangle \\
& +\left\langle q_{i}\left(t_{1}\right) q_{l}\left(t_{3}\right)\right\rangle\left\langle q_{k}\left(t_{2}\right) p_{m}\left(t_{4}\right)\right\rangle \\
& +\left\langle q_{i}\left(t_{1}\right) p_{m}\left(t_{4}\right)\right\rangle\left\langle q_{k}\left(t_{2}\right) q_{l}\left(t_{3}\right)\right\rangle
\end{aligned}
$$

\section{APPENDIX 2: CORRELATIONS IN A SYSTEM OF COUPLED QUANTUM OSCILLATORS}

The system of oscillators is described by the Hamiltonian

$$
H=\frac{1}{2} \sum_{j} p_{i}^{2}+\frac{1}{2} \sum_{j, k} A_{j k} q_{i} q_{k},
$$

where the operators $q_{i}$ and $p_{i}$ satisfy the commutation relations

$$
\left[q_{i}, p_{k}\right]=i \hbar \delta_{i k}, \quad\left[q_{i}, q_{k}\right]=\left[p_{i}, p_{k}\right]=0 .
$$

The expectation value of any operator $F$ with respect to the canonical ensemble at temperature $T$ is defined by

$$
\langle F\rangle=\operatorname{Tr}\left\{F e^{-\beta H}\right\} / \operatorname{Tr}\left\{e^{-\beta H}\right\},
$$

where the trace operation is in the space of the eigenfunctions of the Hamiltonian operator. We are interested mainly in the case where the operator $F$ is a product of $q$ 's and $p$ 's.

As a first step in the evaluation of such traces, consider the eigenvalues and eigenvectors of the matrix A:

$$
\sum_{k} A_{i k} \xi_{k}^{(s)}=\omega_{s}^{2} \xi_{j}^{(s)} .
$$

The eigenvectors are assumed normalized so that

$$
\sum_{j} \xi_{i}^{(s)} \xi_{i}^{(r)}=\delta_{s r}, \quad \sum_{r} \xi_{i}^{(r)} \xi_{k}^{(r)}=\delta_{j k} .
$$

We now introduce the operators

$$
\begin{aligned}
& a_{s}=\left(2 \hbar \omega_{s}\right)^{-\frac{1}{s}} \sum_{i} \xi_{i}^{(s)}\left(p_{i}-i \omega_{\triangleleft} q_{i}\right), \\
& a_{s}^{*}=\left(2 \hbar \omega_{s}\right)^{-\frac{1}{3}} \sum_{i} \xi_{i}^{(s)}\left(p_{i}+i \omega_{s} q_{i}\right) .
\end{aligned}
$$

The commutation relations for these operators follow from (2) and (5). We find

$$
\left[a_{s}, a_{r}^{*}\right]=\delta_{s r}, \quad\left[a_{s}, a_{r}\right]=\left[a_{s}^{*}, a_{r}^{*}\right]=0 .
$$

The inversion of (6) is readily accomplished using the relations (5). We find

$$
\begin{aligned}
& q_{i}=i \sum_{s} \xi_{i}^{(s)}\left(\frac{\hbar}{2 \omega_{s}}\right)^{\frac{1}{3}}\left(a_{s}-a_{s}^{*}\right), \\
& p_{i}=\sum_{s} \xi_{i}^{(s)}\left(\frac{\hbar \omega_{s}}{2}\right)^{\frac{1}{2}}\left(a_{s}+a_{s}^{*}\right) .
\end{aligned}
$$

Inserting these expressions in the Hamiltonian (1) and using the relations (4) and (5) we find

$$
H=\sum_{s} \hbar \omega_{s}\left(a_{s}^{*} a_{s}+\frac{1}{2}\right) .
$$

The operator $a_{s}^{*} a_{\mathrm{s}}$ is the number operator for the $s$ th normal mode; its eigenvalues are the nonnegative integers. The operator $a_{0}$ is the step-down (annihilation) operator, and the operator $a^{*}$ is the step-up (creation) operator; they have matrix elements only between eigenstates of the number operator which differ by unity. ${ }^{15}$

The evaluation of the expectation value (3) when $F$ is a product of the $a$ 's and $a^{*}$ 's is straightforward. Clearly, the only nonvanishing expectation values are for products containing an equal number of $a^{* \prime} s$ and $a$ 's. The simplest of these are the pair expectation values. Thus

$$
\begin{aligned}
\left\langle a_{s}^{*} a_{r}\right\rangle & =\delta_{s r} \operatorname{Tr}\left\{a_{s}^{*} a_{s} e^{-\beta H}\right\} / \operatorname{Tr}\left\{e^{-\beta H}\right\} \\
& =\delta_{s r} \frac{\sum_{n=0}^{\infty} n \exp \left[-\frac{\hbar \omega_{s}}{k T}\left(n+\frac{1}{2}\right)\right]}{\sum_{n=0}^{\infty} \exp \left[-\frac{\hbar \omega_{s}}{k T}\left(n+\frac{1}{2}\right)\right]} \\
& =\delta_{s r}\left[\exp \left(\frac{\hbar \omega_{s}}{k T}\right)-1\right]^{-1}
\end{aligned}
$$

or

$$
\left.\left\langle a_{s}^{*} a_{r}\right\rangle=\frac{1}{2} \delta_{s r}\left[\operatorname{coth} \hbar \omega_{s} / 2 k T\right)-1\right] .
$$

Using the commutation relation (7), we have

$$
\left\langle a_{s} a_{r}^{*}\right\rangle=\frac{1}{2} \delta_{s r}\left[\operatorname{coth}\left(\hbar \omega_{s} / 2 k T\right)+1\right] .
$$

The results for higher products are summarized by the following rule: The expectation value of a product of $a$ 's and $a^{* \prime} \mathrm{s}$ is equal to the sum of products of pair expectation values, the sum being over all possible pairings with the order of each pair preserved.

For example:

$$
\left\langle a_{s}^{*} a_{r} a_{u} a_{v}^{*}\right\rangle=\left\langle a_{s}^{*} a_{r}\right\rangle\left\langle a_{u} a_{v}^{*}\right\rangle+\left\langle a_{s}^{*} a_{u}\right\rangle\left\langle a_{r} a_{v}^{*}\right\rangle .
$$

We do not prove this rule here, since the most convincing demonstration is by example.

We turn now to the consideration of expectation

${ }^{15}$ These properties of the operators are discussed in many textbooks on quantum mechanics. See, e.g., A. Messiah, Quantum Mechanics (North-Holland Publishing Company, Amsterdam, 1961), Chap. 12. 
values of products of $q$ 's and $p$ 's. Because of the linear relations (8) we have the same rule for these expectations:

The expectation value of an odd number of $q$ 's and $p$ 's vanishes. The expectation value of an even number of $q$ 's and $p$ 's is equal to the sum of products of pair expectation values, the sum being over all pairings which preserve the order of the pair.

For example:

$\left\langle q_{i} q_{i} p_{k} q_{l}\right\rangle=\left\langle q_{i} q_{i}\right\rangle\left\langle p_{k} p_{l}\right\rangle+\left\langle q_{i} p_{k}\right\rangle\left\langle q_{i} q_{l}\right\rangle+\left\langle q_{i} q_{l}\right\rangle\left\langle q_{i} q_{k}\right\rangle$.

The pair correlations are readily obtained from (10) and (11), using (8). Thus

$$
\left\langle q_{i} q_{k}\right\rangle=\sum_{s} \frac{\hbar}{2 \omega_{s}} \operatorname{coth} \frac{\hbar \omega_{s}}{2 k T} \xi_{i}^{(s)} \xi_{k}^{(s)} .
$$

Using (4) we see this can be written

$$
\left\langle q_{i} q_{k}\right\rangle=\left\|\frac{\hbar}{2 \mathbf{A}^{1}} \operatorname{coth} \frac{\hbar \mathbf{A}^{\frac{1}{2}}}{2 k T}\right\|_{j k} .
$$

Similarly, we find

$$
\begin{aligned}
& \left\langle p_{j} p_{k}\right\rangle=\left\|\frac{1}{2} \hbar \mathrm{A}^{\frac{1}{2}} \operatorname{coth}\left(\hbar \mathrm{A}^{\frac{1}{3}} / 2 k T\right)\right\|_{i k}, \\
& \left\langle q_{i} p_{k}\right\rangle=-\left\langle p_{i} q_{k}\right\rangle=\frac{1}{2} i \hbar \delta_{j k} .
\end{aligned}
$$

Consider now the time-dependent correlation functions in which the operators at time $t$ are expressed in terms of the initial operators through the relations (4), considered here as formal solutions of the Heisenberg equations of motion. Again we have the rule that correlations of an odd number of operators vanish while a correlation of an even number of operators is equal to a sum of products of pair correlations, the sum being over all pairings which preserve the order of the pair. For example,

$$
\begin{aligned}
\left\langle q_{i}\left(t_{1}\right) q_{k}\left(t_{2}\right) p_{l}\left(t_{3}\right) q_{m}\left(t_{4}\right)\right\rangle & =\left\langle q_{i}\left(t_{1}\right) q_{k}\left(t_{2}\right)\right\rangle\left\langle p_{l}\left(t_{3}\right) q_{m}\left(t_{4}\right)\right\rangle \\
& +\left\langle q_{i}\left(t_{1}\right) p_{l}\left(t_{3}\right)\right\rangle\left\langle q_{k}\left(t_{2}\right) q_{m}\left(t_{4}\right)\right\rangle \\
& +\left\langle q_{i}\left(t_{1}\right) q_{m}\left(t_{4}\right)\right\rangle\left\langle q_{k}\left(t_{2}\right) p_{l}\left(t_{3}\right)\right\rangle .
\end{aligned}
$$

The pair correlations are

$\left\langle p_{i}(t) p_{k}(t+\tau)\right\rangle$

$=\left\|\frac{1}{2} \hbar \mathrm{A}^{\frac{1}{2}}\left[\operatorname{coth}\left(\hbar \mathrm{A}^{\frac{1}{2}} / 2 k T\right) \cos \mathrm{A}^{\frac{1}{2}} \tau+i \sin \mathrm{A}^{\frac{1}{2}} \tau\right]\right\|_{i k}$,

$\left\langle q_{i}(t) q_{k}(t+\tau)\right\rangle$

$=\left\|\frac{1}{2} \hbar \mathrm{A}^{-\frac{1}{j}}\left[\operatorname{coth}\left(\hbar \mathrm{A}^{\frac{1}{2}} / 2 k T\right) \cos \mathrm{A}^{\frac{1}{t}} \tau+i \sin \mathrm{A}^{\frac{1}{t}} \tau\right]\right\|_{i k}$,

$\left\langle q_{i}(t) p_{k}(t+\tau)\right\rangle$

$=\left\|\frac{1}{2} \hbar\left[-\operatorname{coth}\left(\hbar \mathrm{A}^{\frac{1}{t}} / 2 k T\right) \sin \mathrm{A}^{\frac{1}{2}} \tau+i \cos \mathrm{A}^{\frac{1}{2}} \tau\right]\right\|_{i k}$.

The derivation of these expressions goes exactly as the derivation of the corresponding classical correlations, obtained in Appendix 1, but using the expressions (12), (13), and (14) for the initial expectation values. 